\title{
On the Extrapolation of CFD Results for Smoke and Heat Control in Reduced-Scale Set-Ups to Full Scale: Atrium Configuration
}

\author{
Nele Tilley ${ }^{1}$, Pieter Rauwoens ${ }^{2}$, Dieter Fauconnier ${ }^{1}$ and Bart Merci ${ }^{1}$ \\ ${ }^{1}$ Ghent University, Department of Flow, Heat and Combustion Mechanics, \\ Sint-Pietersnieuwstraat 41, B-9000 Ghent, Belgium \\ ${ }^{2}$ Ghent University, Department of Civil Engineering, \\ Technology Park 904, B-9052 Ghent, Belgium
}

Corr. author: Bart.Merci@UGent.be, Tel: +32 926433 14, Fax: +32 92643575

\begin{abstract}
It is common practice to use reduced-scale experiments to develop formulae for the design of smoke and heat exhaust ventilation systems. Implicitly, up-scaling of results is assumed justified. A similar approach can be adopted with numerical simulations, i.e. a reduced-scale setup can be up-scaled to compare results to full-scale observations. However, both in numerical simulations and in experiments, scaling must be done in a proper way. The classical method for up-scaling results obtained in fire related experiments is based on preservation of the Froude number only. The present paper, focusing on the up-scaling of results by means of a series of CFD simulations of fire-induced flows in an atrium configuration, confirms this to be justified as long as the flows in both the reduced-scale and full-scale configurations are sufficiently turbulent. If this is not the case, it is illustrated that other dimensionless numbers must also be preserved in scaling, in addition to the Froude number.
\end{abstract}




\section{Introduction}

Atria are a commonly encountered architectural type of buildings. They are often found in shopping malls, hotels and office buildings. When fire occurs in a room adjacent to the atrium, a smoke plume can emerge and spill into the atrium. This spill plume then rises to the ceiling of the atrium, to form a smoke layer underneath (Fig.1).

A smoke and heat control system can be designed in the atrium to extract smoke at ceiling level. In the past, several authors [1-9] developed formulae to calculate the required smoke mass flow rate $(\dot{m})$ to be extracted at the ceiling of the atrium, in order to ensure a certain smoke free height $\left(z_{s}\right)$ above the spill edge. The (convective) fire heat release rate $\left(\dot{Q}_{\text {conv }}\right)$ and a length scale parameter of the atrium (typically a width $W$ ) appear in all these formulae.

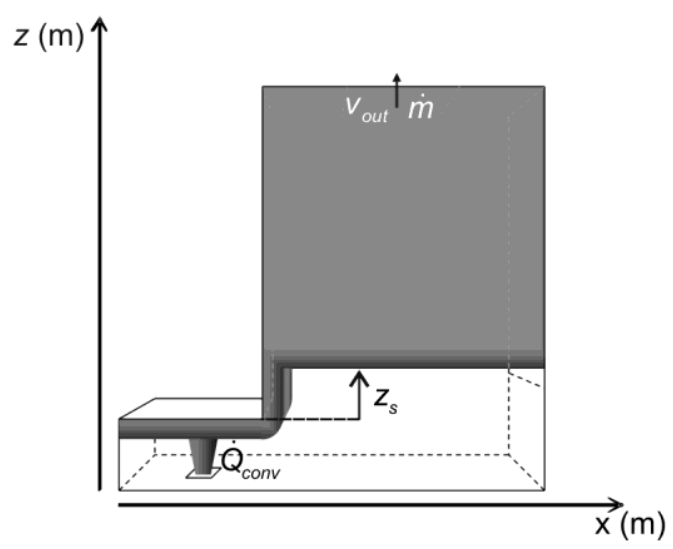

Figure 1. Schematic representation of smoke filling in an atrium configuration (fire in adjacent room).

These expressions rely on reduced-scale experiments. Being less expensive, safer to control and easier to handle for a parameter variation study, and requiring less space, reduced-scale experiments are indeed usually preferred over full-scale experiments. However, for reducedscale data formulae to be used in full-scale configurations, proper scaling must be applied. This is based on the preservation of the Froude number, which is justified as long as the flow is sufficiently turbulent. Keeping the Froude number constant, together with other 
dimensionless numbers like e.g. the Reynolds number, proves to be very hard to accomplish in practice in experiments. The reduced-scale experimental setup should then be in nonatmospheric conditions (different gravity, and/or different "air" viscosity), which is very difficult and expensive to obtain. Therefore, only the Froude number is really kept constant in the scaling procedure.

With CFD (Computational Fluid Dynamics) calculations, parameter variation is easier. In the present paper, we perform a series of numerical simulations. The intention is to illustrate the effect of reduced levels of turbulence (in reduced-scale set-ups) in Froude number based scaling of results.

In the present paper, FDS (Fire Dynamics Simulator, version 5.5.3 [10, 11]) is used as CFD code. Therefore the governing equations are written below as presented in the FDS manual. However, conclusions on dimensionless numbers are valid for the equations to be solved in any CFD program. The three basic governing flow equations (conservation of mass and total momentum, and transport of sensible enthalpy), in their instantaneous form, serve as starting point. The derivation of the important dimensionless numbers is briefly described. Such theoretical studies have already been reported in text books (e.g. [12]). The novelty in the paper at hand is a follows:

1. The limitations of Froude number scaling are illustrated by means of a set of CFD simulations of smoke and heat control in an atrium configuration, using a Large Eddy Simulation (LES) approach with the standard Smagorinsky subgrid scale model [13] to model turbulence.

2. It is confirmed that CFD results remain similar if, along with the Froude number, the Reynolds number is preserved. Preserving the Froude number alone is confirmed not to be sufficient when the flow becomes weakly turbulent. 


\section{Governing equations - scaling laws}

The most important scaling features are briefly mentioned, as they are relevant in the discussion of the results. For a more extensive theoretical discussion, the reader is referred to e.g. [12].

\section{$\underline{2.1 \text { Instantaneous equations and dimensionless numbers }}$}

\section{Conservation of mass}

For a single phase flow, this reads:

$\frac{\partial \rho}{\partial t}+\nabla \cdot \rho \mathbf{v}=0$

Stating that $\frac{\rho}{t} \sim \frac{\rho v}{L}$ implies

$t \sim L / v$.

In time-dependent scaled simulations, it is important to use this scaling factor to compare different instantaneous flow fields, with different characteristic length scales L and velocity scales $\mathrm{v}$ at corresponding time levels. In other words, results at time $t_{r}$ in reduced-scale, must be compared to results at time $t_{f}=\left(L_{f} v_{r}\right) /\left(L_{r} v_{f}\right) t_{r}$ in the full-scale setup. Obviously, instantaneous LES results are of limited value. Yet, instantaneous results are shown below in the quasi steady-state range for comparison purposes. The relation $t_{f}=\left(L_{f} v_{r}\right) /\left(L_{r} v_{f}\right) t_{r}$ implies that it takes longer to reach quasi steady-state conditions in larger configurations.

\section{Conservation of momentum}

$$
\begin{aligned}
& \frac{\partial}{\partial t}(\rho \mathbf{v})+\nabla \cdot \rho \mathbf{v} \mathbf{v}=-\nabla\left(p+\rho_{0} g \mathbf{z}\right)+\left(\rho_{0}-\rho\right) \mathbf{g}+\nabla \cdot \boldsymbol{\tau} \\
& \text { with } \boldsymbol{\tau}=\mu\left(2 \mathbf{S}-\frac{2}{3} \boldsymbol{\delta}(\nabla \cdot \mathbf{v})\right) \\
& \qquad S_{i j}=\frac{1}{2}\left(\frac{\partial v_{i}}{\partial x_{j}}+\frac{\partial v_{j}}{\partial x_{i}}\right)
\end{aligned}
$$


Equation (3) reveals that $\frac{\rho v}{t} \sim \frac{\rho v^{2}}{L} \sim \frac{p}{L} \sim \Delta \rho g \sim \mu \frac{v}{L^{2}}$, resulting in two important dimensionless numbers:

- The Froude number, comparing inertia and buoyancy forces, which is important in flows driven by natural convection: $\mathrm{Fr}=v \sqrt{\rho} / \sqrt{g L \Delta \rho}$. As natural convection is predominant in the test case at hand, keeping Fr constant is the logical first choice, which is also followed in the derivation of the one-line formulae from reduced-scale experiments as presented in [1-9]. Maintaining temperatures equal in original and scaled setups, the Froude number is typically simplified to:

$$
\operatorname{Fr}=\frac{v}{\sqrt{g L}}
$$

This definition for Fr is used below.

- The Reynolds number, comparing inertia and viscous forces, which is used primarily in flows with forced convection:

$$
\operatorname{Re}=\frac{\rho v L}{\mu}=\frac{v L}{v}
$$

In both Reynolds and Froude number, $L$ is a representative distance length scale.

Transport of sensible enthalpy

$$
\frac{\partial}{\partial t}\left(\rho h_{s}\right)+\nabla \cdot \rho h_{s} \mathbf{v}=\frac{\partial p}{\partial t}+\dot{q}^{\prime \prime \prime}-\nabla \cdot \dot{\mathbf{q}}^{\prime \prime}+\tau_{i j} \nabla \mathbf{v}
$$

With $\dot{\mathbf{q}}^{\prime \prime}=-k \nabla T-\sum_{a} h_{s, a} \rho D_{a} \nabla Y_{a}+\dot{\mathbf{q}}_{r}^{\prime \prime}$

$$
h_{s}=\sum_{a} Y_{a} h_{s, a}=\sum_{a}\left(Y_{a} \int_{T_{0}}^{T} c_{p, a} T^{\prime} d T^{\prime}\right)
$$

As the equation for transport of sensible enthalpy is written in the form as used in FDS simulations, the reader is referred to the FDS manuals $[10,11]$ for more information on the different terms in this equation. 
Eq. (8) leads to: $\frac{\rho c_{p} T}{t} \sim \frac{\rho c_{p} T v}{L} \sim \frac{p}{t} \sim \frac{\dot{Q}}{L^{3}} \sim \frac{k T}{L^{2}} \sim \frac{\rho c_{p} T D}{L^{2}} \sim \mu \frac{v^{2}}{L^{2}}$

- From transport of sensible enthalpy, the velocity scales as

$$
v \sim \frac{\dot{Q}}{L^{2} \rho c_{p} \Delta T} .
$$

- The ratio of kinematic viscosity (or 'momentum' diffusivity) and thermal diffusivity is the Prandtl number:

$$
\operatorname{Pr}=\frac{v}{\alpha}=\frac{\mu c_{p}}{k}
$$

- Similarly, the Schmidt number expresses the ratio of momentum diffusivity and mass diffusivity:

$\mathrm{Sc}=\frac{v}{D}$

- A final important dimensionless number is the Rayleigh number, a combination of $\operatorname{Re}, \operatorname{Pr}$ and Fr. The Rayleigh number, primarily used for natural convection, is:

$$
\mathrm{Ra}=\frac{\operatorname{Re}^{2} \operatorname{Pr}}{\operatorname{Fr}^{2}}=g \frac{L^{3}}{v \alpha} \frac{\Delta \rho}{\rho} .
$$

\subsection{Influence of numerical aspects and modeling}

The above presented dimensionless numbers are based on molecular thermo-physical properties. In CFD, Direct Numerical Simulation of the flow, in which all turbulent scales are fully resolved down to the Kolmogorov scale, i.e. the smallest turbulent scale, is prohibitively expensive due to the fine grid requirements. Therefore, the flow field is typically resolved on a much coarser mesh, making use of models to describe the influence of small unresolved turbulent scales on the large resolved scales. In LES, the equation for conservation of momentum is filtered, using a density weighting or Favre averaging, yielding 
$\frac{\partial(\bar{\rho} \tilde{\mathbf{v}})}{\partial t}+\nabla \cdot(\bar{\rho} \tilde{\mathbf{v}} \tilde{\mathbf{v}})=-\nabla\left(\bar{p}+\rho_{0} g \overline{\mathbf{z}}\right)+\left(\rho_{0}-\bar{\rho}\right) \mathbf{g}+\nabla\left(\overline{\boldsymbol{\tau}}-\tau^{*}\right)$

$\tau^{*}$ denotes the residual stress tensor and represents the effect of the unresolved scales on the resolved ones. The energy equation is treated in a similar manner. The residual stress tensor is typically modeled by means of an eddy viscosity model. As such, turbulent viscosity and diffusivity are introduced, contributing in e.g. the Reynolds-number. In FDS, by default the standard Smagorinsky turbulence model is used. In this model, the turbulent dynamic viscosity is defined, as:

$\mu_{T}=\bar{\rho}\left(C_{s} \Delta\right)^{2}|\tilde{\mathbf{S}}|$,

with $\Delta$ the grid cell size. Although FDS uses an adjusted modelling approach, the conclusions derived below remain valid, so that for the sake of the present paper it is not necessary to go into more detail on the FDS approach. It is interesting to note that this turbulent viscosity globally scales as:

$v_{T} \sim\left(\frac{\Delta}{L}\right)^{2} v L$

When the ratio $\Delta / \mathrm{L}$ goes to 0 , the turbulent viscosity decreases and the simulation evolves towards a DNS simulation.

If the same number of mesh cells is used in the reduced-scale and full-scale setup, the cell size scales proportional to the configuration dimensions, the ratio $\Delta / \mathrm{L}$ is constant and the turbulent viscosity then scales as

$v_{T} \sim v L$

This reveals that the modelled turbulence scales properly in the sense that the Reynolds number based on turbulent viscosity,

$\operatorname{Re}_{T}=\frac{v L}{v_{T}}$ 
is automatically preserved. Indeed, the filtered equation for conservation of momentum (Eq. 15) yields $\frac{\rho v}{t} \square \frac{\rho v^{2}}{L} \square \frac{p}{L} \square \Delta \rho g \square \mu \frac{v}{L^{2}} \square \mu_{T} \frac{v}{L^{2}}$. Thus, using eq. (18) implies that the turbulent Reynolds number is automatically preserved when using the Smagorinsky LES model and when the grid cell size is scaled proportional to the configuration dimensions (which was the assumption leading to eq. (18)).

In a similar way, the filtered equation for conservation of energy can be derived to show that a turbulent Pr number is automatically preserved when keeping the Fr number constant.

\subsection{Scaling of experiments}

When scaling up experimental configurations, obviously no modeling effects are taken into account. In experimental test cases, the results are what they are, by definition according to reality (which is measured up to a certain accuracy level).

However, it is important to recall the physics of scaling when dealing with experiments. Especially the difference between laminar and turbulent flow is important. The distinction laminar/turbulent relies on the Reynolds number (Eq. (7)) or the Rayleigh number (Eq. (14)). If the same fluid is used in reduced-scale and full-scale setup, temperatures are kept equal and Fr is constant, the Reynolds number scales as $\operatorname{Re} \square v L \square L^{3 / 2}$, while Ra scales as $R a \square L^{3}$. As a consequence, there is a potential danger that a laminar, or weakly turbulent, reduced-scale experiment is scaled up, via the Froude-number, to a strongly turbulent full-scale configuration. In this case, the two configurations need not give similar results.

It is well-known that only when both reduced-scale and full-scale setups have a turbulent flow behavior (Reynolds and Rayleigh numbers are high, say $\operatorname{Re}>10^{4}$ and $\mathrm{Ra}>10^{9}$ ), self-similar behavior can be expected.

This will now be discussed more extensively in the next section. 


\section{Results}

\section{$\underline{3.1 \text { Set-up of simulations }}$}

A number of CFD simulations have been performed to investigate the effects of scaling. The geometric configuration is always kept the same, similar to the atrium configuration in the experiments of [8]. In the 'basic' simulation, the atrium height equals $H_{a}=3.24 \mathrm{~m}$ and the width $W_{s}=0.81 \mathrm{~m}$. These dimensions have been multiplied by a factor 9 in the 'full-scale' configuration $\left(H_{a}=29.16 \mathrm{~m}, W_{s}=7.29 \mathrm{~m}\right)$. The grid consists of 43200 cubic cells in the adjacent room and 518400 cells of the same size $(2.25 \mathrm{~cm} \times 2.25 \mathrm{~cm} \times 2.25 \mathrm{~cm}$ in the basic simulation) in the atrium.

As the rising spill plume is strongly determined by the width of the smoke layer as it emerges from the adjacent room into the atrium, the atrium width at the spill edge $\left(W_{s}\right)$ is taken as characteristic length scale in the calculation of the Re and Ra numbers.

In scaling the configurations, the Froude number is always preserved. The temperature field has also been kept identical to that in the original simulation. As the height of the smoke layer is defined by application of the N-percent rule to the temperature profile [14], the ratio of this height to the atrium height will then also be preserved. Also, $c_{p}$ values and densities remain practically unchanged. Gravity and ambient molecular dynamic viscosity can be changed. The Reynolds number (Eq. 7) indeed provides the scaling for kinematic viscosity:

$v \square v_{\text {out }} W_{s}$,

with $v_{\text {out }}$ a velocity defined below.

The turbulent Schmidt and Prandtl numbers are kept constant.

Keeping the Froude number (Eq. (6)) constant, the outlet velocity scales as

$v_{\text {out }} \square \sqrt{g W_{s}}$

As boundary conditions, a constant velocity $v_{\text {out }}$ is imposed over the entire surface of the opening in the atrium ceiling, resembling mechanical smoke extraction. The walls of the 
atrium are modeled as adiabatic, so that issues on thermal boundary conditions are avoided in the scaling procedure.

To represent the fire, a flow of hot air is inserted as heat source in the adjacent room of the atrium. As such, issues in combustion modeling, which are not relevant for the sake of the present paper, are avoided.

The heat release rate of this flow is calculated as

$\dot{Q}_{\text {conv }}=A_{F} \cdot u \cdot \rho_{\text {in }} \cdot\left(T_{\text {in }}-T_{0}\right) \cdot c_{p}$

In the 'basic' simulation, the heat release rate is $4.34 \mathrm{~kW}$, with $A_{F}=0.0506 \mathrm{~m}^{2}, T_{\text {in }}=510^{\circ} \mathrm{C}, u$ $=0.39 \mathrm{~m} / \mathrm{s}$ and $\rho_{\text {in }}=0.45 \mathrm{~kg} / \mathrm{m}^{3}$.

The equivalence of velocity (Eq. 11) leads to the proper scaling law for the heat release rate of the fire:

$\dot{Q} \square v_{\text {out }} W_{s}^{2}$

\section{Simulations}

Eight simulations are analyzed in this paper. Table 1 summarizes the dimensionless numbers. $\mathrm{Re}, \mathrm{Ra}$ and Fr numbers have been determined using $v_{\text {out }}$ and $W_{s}$ The numbers show that the flow is fully turbulent $\left(\operatorname{Re}>10^{4}\right.$ and $\left.\operatorname{Ra}>10^{9}\right)$ in all simulations except for 7 and 8 (where the flow is much less turbulent or not even turbulent at all). The use of a standard Smagorinsky turbulence model in an LES simulation of a weakly turbulent or non-turbulent flow is questionable when a high level of accuracy is pursued. However, although this deserves some attention in assessing the agreement of simulation results to experimental data for such flows, to the best of the authors' knowledge this issue has not been addressed yet in the fire science research community and is not considered as crucial for the sake of the present paper, discussing the limits of Froude number scaling.

The actually imposed values are presented in Table 2 . While simulations 1, 3 and 5 concern a reduced-scale atrium $\left(W_{s}=0.81 \mathrm{~m}\right)$, simulations 2,4 and 6 represent a more realistic atrium 
$\left(W_{s}=7.29 \mathrm{~m}\right)$. Simulations 1 and 2 are both in regular atmospheric conditions (gravity and viscosity), and thus represent scaling from reduced-scale experiment to full-scale configuration. Note that in simulation 2 , the Re and Ra numbers are not preserved with respect to simulation 1 .

In simulations 3 and 4, only the ambient molecular viscosity is modified. As Reynolds and Rayleigh numbers are high, the flow is sufficiently turbulent and a variation in the molecular viscosity is not expected to affect the result.

In simulations 5 and 6, the gravity is artificially reduced in the simulations. With Eq. (20), (21) and (23), the other initial parameters can be calculated to ensure that $\mathrm{Re}, \mathrm{Ra}$ and Fr are preserved with respect to simulation 1. This kind of scaling is hardly ever possible in experiments, as changing gravity is very difficult to accomplish. Yet, it is an interesting numerical test case to perform with CFD.

Simulation 7 and 8 again concern the reduced-scale atrium, with only a change in molecular viscosity. Again, it is scaled by Froude scaling, so the Fr number is preserved, but $\mathrm{Re}$ and $\mathrm{Ra}$ are not. In fact, Re and Ra are lower than the turbulence limit in these simulations. 


\begin{tabular}{|c|c|c|c|c|c|c|c|}
\hline $\mathbf{n}^{\mathbf{0}}$ & $\mathbf{R e}$ & $\mathbf{R a}$ & $\boldsymbol{Q}$ & $\boldsymbol{\mu}$ & $\boldsymbol{u}_{\text {out }}$ & $\boldsymbol{g}$ & $\boldsymbol{W}_{\boldsymbol{s}}$ \\
\hline 1 & $\operatorname{Re}_{1}$ & $\mathrm{Ra}_{1}$ & $Q_{1}$ & $\mu_{1}$ & $u_{1}$ & $g_{1}$ & $W_{s, 1}$ \\
\hline 2 & $a^{3 / 2} \operatorname{Re}_{1}$ & $a^{3} \mathrm{Ra}_{1}$ & $a^{5 / 2} Q_{1}$ & $\mu_{1}$ & $a^{1 / 2} u_{1}$ & $g_{1}$ & $a W_{s, 1}$ \\
\hline 3 & $a^{3 / 2} \operatorname{Re}_{1}$ & $a^{3} \mathrm{Ra}_{1}$ & $Q_{1}$ & $a^{-3 / 2} \mu_{1}$ & $u_{1}$ & $g_{1}$ & $W_{s, 1}$ \\
\hline 4 & $\operatorname{Re}_{1}$ & $\operatorname{Ra}_{1}$ & $a^{5 / 2} Q_{1}$ & $a^{3 / 2} \mu_{1}$ & $a^{1 / 2} u_{1}$ & $g_{1}$ & $a W_{s, 1}$ \\
\hline 5 & $\operatorname{Re}_{1}$ & $\operatorname{Ra}_{1}$ & $a^{-1 / 2} Q_{1}$ & $a^{-1 / 2} \mu_{1}$ & $a^{-1 / 2} u_{1}$ & $a^{-1} g_{1}$ & $W_{s, 1}$ \\
\hline 6 & $\operatorname{Re}_{1}$ & $\operatorname{Ra}_{1}$ & $a^{2} Q_{1}$ & $a \mu_{1}$ & $u_{1}$ & $a^{-1} g_{1}$ & $a W_{s, 1}$ \\
\hline 7 & $a^{-3 / 2} \operatorname{Re}_{1}$ & $a^{-3} \operatorname{Ra}_{1}$ & $Q_{1}$ & $a^{3 / 2} \mu_{1}$ & $u_{1}$ & $g_{1}$ & $W_{s, 1}$ \\
\hline 8 & $a^{-1} \operatorname{Re}_{1}$ & $a^{-2} \operatorname{Ra}_{1}$ & $Q_{1}$ & $a \mu_{1}$ & $u_{1}$ & $g_{1}$ & $W_{s, 1}$ \\
\hline
\end{tabular}

Table 1. Scaling of the imposed parameters in the eight simulations. Scaling factor $a=9$, and constant Froude Number Fr $\left(\operatorname{Re}_{1}=16000 ; \mathrm{Ra}_{1}=2.210^{9} ; \mathrm{Fr}=0.106\right) . \mathrm{RS}=$ reduced-scale; FS $=$ full-scale. All simulations are fully turbulent ( 3 and 4 with changed viscosity), except for 7 and 8 . In simulations 5 and 6 , the gravity is changed.

\begin{tabular}{|c|c|c|c|c|c|}
\hline $\boldsymbol{Q}_{\mathbf{1}}$ & $\boldsymbol{\mu}_{\mathbf{1}}$ & $\boldsymbol{u}_{\text {out } \mathbf{1}}$ & $\boldsymbol{g}_{\mathbf{1}}$ & $\boldsymbol{H}_{\boldsymbol{a}, \mathbf{1}}$ & $\boldsymbol{W}_{\boldsymbol{s}, \mathbf{1}}$ \\
\hline $\mathrm{kW}$ & $\mathrm{kg} / \mathrm{sm}$ & $\mathrm{m} / \mathrm{s}$ & $\mathrm{m} / \mathrm{s}^{2}$ & $\mathrm{~m}$ & $\mathrm{~m}$ \\
\hline 4.34 & $18 \cdot 10^{-6}$ & 0.3 & 9.81 & 3.24 & 1.19 \\
\hline
\end{tabular}

Table 2. Initial values of the imposed parameters in "basic" simulation 1. 


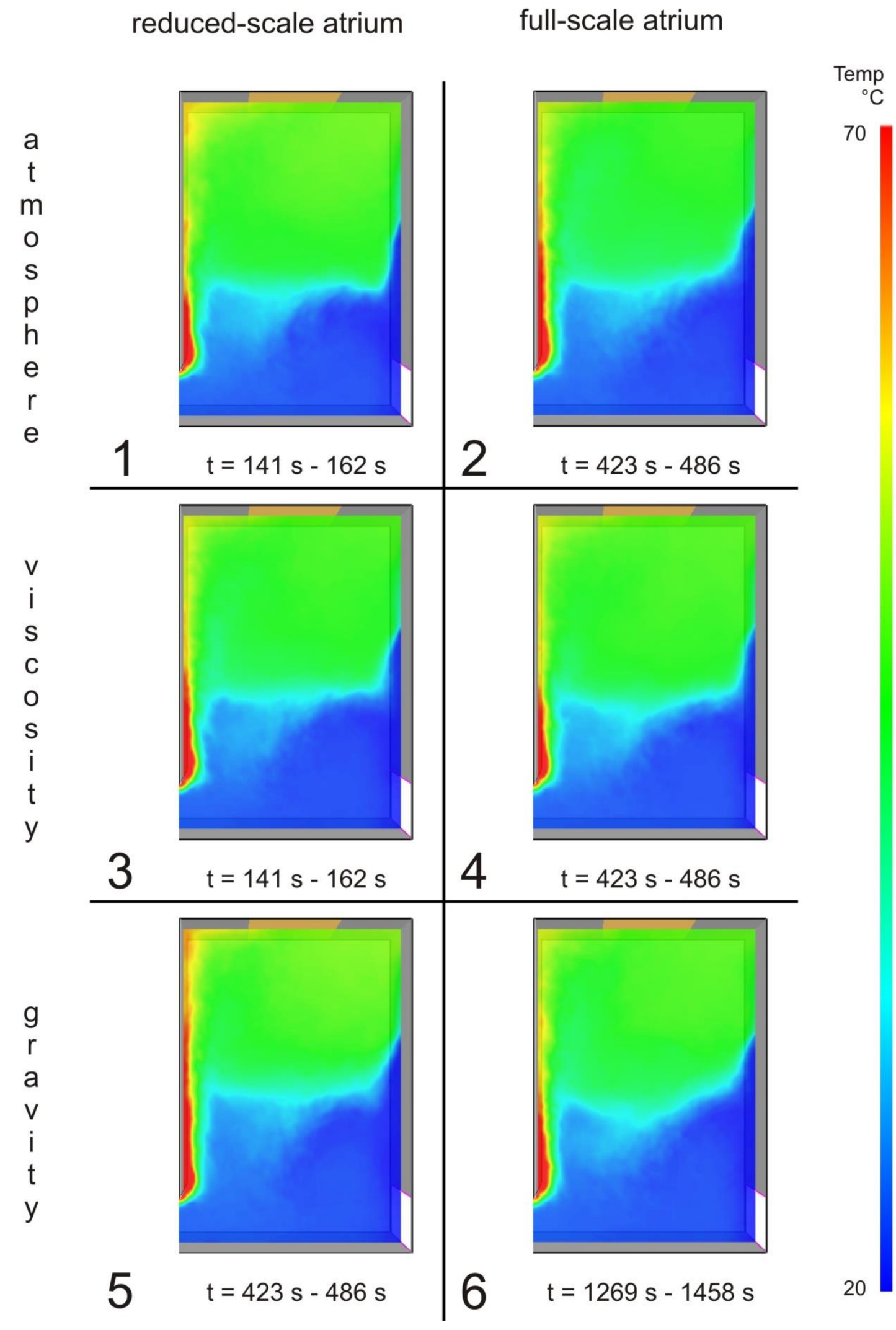

Figure 2. Averaged temperature fields in the vertical symmetry plane at corresponding times of simulations 1-6. 


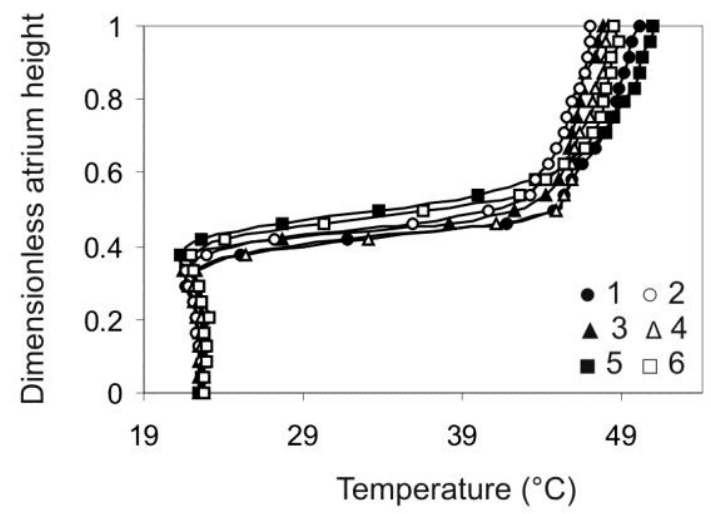

Figure 3. Temperature simulation data on a vertical line in the atrium.

Figures 2 and 3 show the results for temperature in simulations 1 through 6 . While Fig. 2 shows an averaged quasi-steady state temperature field in the vertical symmetry plane for each simulation, Fig. 3 displays the temperature profile on a vertical line in the atrium in all six simulations together. Both figures show that the temperature fields all agree well with each other. This reveals that, for the test case under study, scaling based on the Froude number alone is allowed, since e.g. in simulations 2 and 3 the Reynolds and Rayleigh numbers are not preserved. This also indicates that the correlations, derived for reduced-scale setups, can be applied to full-scale configurations. It is recalled here that this is usually assumed to only be true when all configurations imply well developed turbulence.

In simulations 7 and 8 , on the other hand, the Re and Ra number are low, resulting in a laminar or transitional flow, respectively. The comparison of simulations 1, 3, 7 and 8 (all in the reduced-scale configuration, the only difference is the molecular viscosity) is displayed in Figures 4 and 5. 


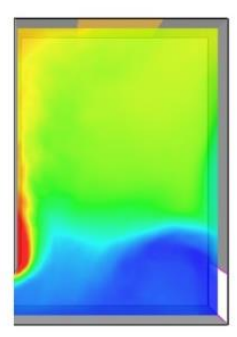

7

$a^{3 / 2} \mu_{1}$

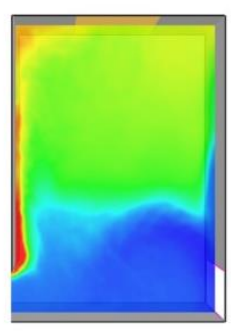

8

a $\mu_{1}$

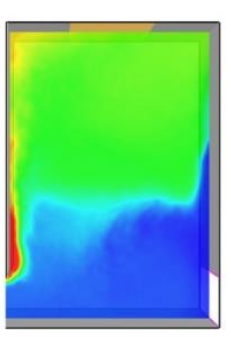

1

$\mu_{1}$

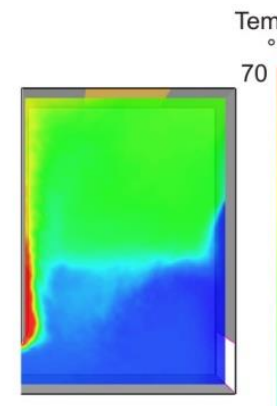

3

$a^{-3 / 2} \mu_{1}$

Figure 4. Averaged temperature fields between $t=141 \mathrm{~s}-162 \mathrm{~s}$ of simulations $7,8,1$ and 3 .

Ordering from left to right: laminar $\rightarrow$ turbulent.

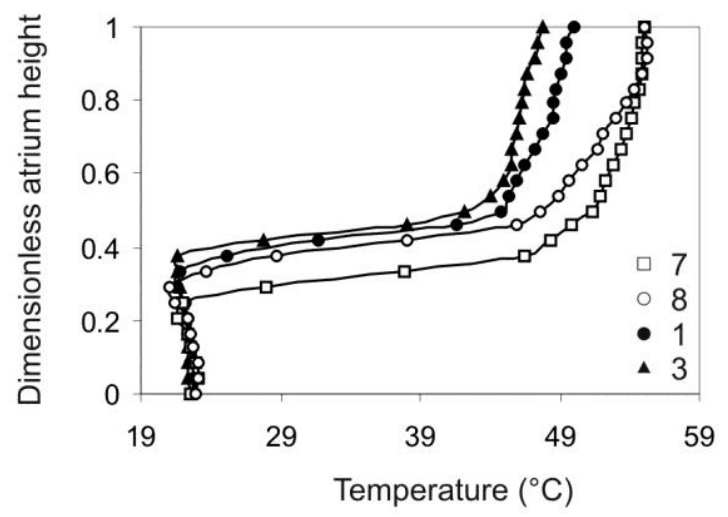

Figure 5. Temperature simulation data on a vertical line in the atrium.

The figures clearly show that the results from simulation 7 are not in agreement with the other ones. The reason is that the molecular thermal diffusivity is higher in case 7 than in the basic case: the molecular viscosity increases and the Prandtl number is preserved. Not only does this affect the entrainment in the region where the smoke turns upward from the horizontal motion into the atrium, it also results in more diffusion of high temperatures into the entire smoke layer. Figure 4 indeed reveals that the high-temperature region is more concentrated near the left atrium wall in the basic case (1) than in case 7. Obviously, this is reflected in Fig. 5. The higher (thermal) diffusivity also leads to a thicker smoke layer than for the basic case: 
the smoke is more widely spread over the atrium. Also the result of simulation 8 still deviates a little from the 'fully' turbulent results of simulations 1 and 3. As the Re and Ra numbers are too low to represent fully developed turbulence, data from these simulations are thus not representative, despite preservation of the Fr number. In other words: such results must not be up-scaled. [Note that the explanation for the discrepancies between simulations 7 or 8 and 1 or 3 is on the basis of molecular viscosity, not by regarding the former simulations as laminar. Indeed: by using the standard Smagorinsky turbulence model and applying a Froude scaling, the turbulence level is always retained (see section 2.2).]

Note that the simulations carried out with higher molecular viscosities are used to address the scaling of numerical simulations, but they are not meant to be conclusive with respect to scaling of experiments. The reason for this is that we adopt a standard Smagorinsky model, from which we know it does not tend to a zero turbulent viscosity in laminar flow regimes. It is therefore possible that an experimental set-up of simulation 7 or 8 is more similar to the base case than the numerical results suggest.

It is interesting to note that simulation 4 has the same molecular viscosity as simulation 7 . Between these two simulations, only the dimensions of the setup have changed, which is the same difference as between simulations 1 and 2. However, in simulations 1 and 2 the flow is fully turbulent and thus the temperature results are in agreement.

In short, the CFD analysis confirms that Froude scaling is only allowed as long as the turbulent flow in the reduced-scale setup is fully developed, and that this is automatically captured in the simulations. 


\section{Conclusion}

Scaling of results, based on dimensionless numbers, has been discussed in the context of CFD simulations of smoke and heat control in an atrium configuration.

The following aspects have been automatically captured and confirmed:

- Scaling based on only the Froude number is justified as long as all configurations display well developed turbulence.

- CFD results remain similar if, along with the Froude number, the Reynolds number is preserved. Preserving the Froude number alone is not to sufficient when the flow becomes weakly turbulent.

- Since similarity is lost if the flow is not sufficiently turbulent and only the Froude number is preserved in the scaling procedure, care must be taken not to operate in too low Reynolds or Rayleigh number ranges when performing model scale studies, be it in 'real' or 'numerical' experiments.

\section{Acknowledgements}

Research funded by a PhD grant of the Institute for the Promotion of Innovation through Science and Technology in Flanders (IWT-Vlaanderen). 


\section{References}

[1] Law, M., "A Note on Smoke Plumes from Fires in Multi-level Shopping Malls”, Fire Safety Journal 10(3): 197-202 (1986).

[2] Thomas, P.H., "On the Upward Movement of Smoke and Related Shopping Mall Problems”, Fire Safety Journal 12(3): 191-203 (1987).

[3] Law, M., "Measurements of Balcony Smoke Flow”, Fire Safety Journal 24(2): 189195 (1995).

[4] NFPA 92B, Smoke Management Systems in Malls, Atria and Large Areas, National Fire Protection Association (2005).

[5] Poreh, M., Morgan, H.P., Marshall, N.R., Harrison, R., "Entrainment by TwoDimensional Spill Plumes”, Fire Safety Journal 30(1): 1-19 (1998).

[6] Thomas, P.H., Morgan, H.P., Marshall, N., "The Spill Plume in Smoke Control Design”, Fire Safety Journal 30(1): 21-46 (1998).

[7] Harrison, R., Spearpoint, M., "Entrainment of air into a balcony spill plume", Journal of Fire Protection Engineering 16: 211-245 (2006).

[8] Poreh, M., Marshall, N.R., Regev, A., "Entrainment by adhered two-dimensional plumes”, Fire Safety Journal 43: 344-350 (2008).

[9] Harrison R, Spearpoint M, Physical scale modeling of adhered spill plume entrainment, Fire Safety J 45: 149-158 (2010)

[10] McGrattan, K., Klein, B., Hostikka, S., Floyd, J., Fire Dynamics Simulator (Ver.5) User's Guide, NIST 1019-5, National Institute of Standards and Technology (2008).

[11] McGrattan K, Hostikka S, Floyd J, Baum H, Rehm R, Mell W, McDermott R, Fire Dynamics Simulator (Version 5) Technical Reference Guide, NIST 1018-5, National Institute of Standards and Technology, 2008 
[12] Quintiere, J., Fundamentals of Fire Phenomena, John Wiley and Sons, Chichester, 2006.

[13] Smagorinsky J, General Circulation Experiments with the Primitive Equations. I. The Basic Experiment, Monthly Weather Review 1963;91:99-164

[14] Cooper LY, Harkleroad M, Quintiere J, Reinkinen W, An Experimental Study of Upper Hot Layer Stratification in Full-Scale Multiroom Fire Scenarios, Journal of Heat Transfer 1982;104:741-749 


\section{Tables}

\section{List of symbols}

$\dot{Q} \quad$ heat release rate $\quad \mathrm{kW}$

$\dot{m} \quad$ mass flow rate $\mathrm{kg} / \mathrm{s}$

$\dot{q} \quad$ heat release rate $\quad \mathrm{kW}$

$A_{F} \quad$ heat source area $\quad \mathrm{m}^{2}$

$\begin{array}{lll}c_{p} & \text { specific heat capacity } \mathrm{J} / \mathrm{kgK}\end{array}$

$C_{S} \quad$ Smagorinsky constant

D mass diffusivity $\mathrm{m}^{2} / \mathrm{s}$

$f_{b} \quad$ external force $\quad \mathrm{N}$

$g \quad$ gravity acceleration $\quad \mathrm{m} / \mathrm{s}^{2}$

$\begin{array}{lll}H_{a} & \text { atrium height } & \mathrm{m}\end{array}$

$\begin{array}{lll}h_{s} & \text { Enthalpy } \quad \mathrm{J} / \mathrm{kg}\end{array}$

$k \quad$ thermal conductivity $\mathrm{kW} / \mathrm{mK}$

L Length $\quad$ m

$N \quad$ number in $N$-percent rule

$p \quad$ Pressure $\quad \mathrm{Pa}$

$S \quad$ rate of strain $1 / \mathrm{s}$

$t \quad$ Time $\mathrm{s}$

$\begin{array}{lll}T & \text { Temperature } & \mathrm{K}\end{array}$

$u \quad$ heat source inlet velocity $\mathrm{m} / \mathrm{s}$

$v \quad$ Velocity $\mathrm{m} / \mathrm{s}$

$W_{s} \quad$ atrium width $\quad \mathrm{m}$

$\begin{array}{lll}x & \text { Length } & \mathrm{m}\end{array}$

Y mass fraction 
$z$

Height

$\Delta$

grid cell size

$\alpha$

$\mu$

v

$\rho$

$\tau$

stress tensor m

$\mathrm{m}$

$\mathrm{m}^{2} / \mathrm{s}$

$\mathrm{kg} / \mathrm{ms}$

$\mathrm{m}^{2} / \mathrm{s}$

$\mathrm{kg} / \mathrm{m}^{3}$

$\mathrm{Pa}$

\section{Subscript}

$\begin{array}{ll}\text { conv } & \text { convective } \\ \text { eff } & \text { Effective } \\ f & \text { full-scale } \\ \text { in } & \text { heat source inlet } \\ r & \text { reduced-scale } \\ T & \text { Turbulent } \\ 0 & \text { ambient conditions }\end{array}$

